\title{
DEMONSTRATION OF FEMTOSECOND-PHASE STABILIZATION IN 2 KM OPTICAL FIBER *
}

\begin{abstract}
J. W. Staples, R. Wilcox and J. M. By
\section{Abstract}

Long-term phase drifts of less than a femtosecond per hour have been demonstrated in a $2 \mathrm{~km}$ length of singlemode optical fiber, stabilized interferometrically at 1530 $\mathrm{nm}$. Recent improvements include a wide-band phase detector that reduces the possibility of fringe jumping due to fast external perturbations of the fiber and locking of the master CW laser wavelength to an atomic absorption line. Mode-locked lasers may be synchronized using two wavelengths of the comb, multiplexed over one fiber, each wavelength individually interferometrically stabilized.
\end{abstract}

\section{INTRODUCTION}

The next generation of accelerators will require supplying timing signals and RF synchronization with differential mode drifts down to the femtosecond level over many hours. In addition, the fast jitter, when pulse-probe experimental lasers are locked together, must also be in the low femtosecond regime.

Single-mode optical fiber operating in the $1550 \mathrm{~nm}$ range, such as developed and used in the telecommunications community, offers multi-GHz bandwidth, very low attenuation over kilometer lengths and immunity from electrical interference. However, fiber is subject to changes in phase and group velocity correlated to temperature variations, is sensitive to acoustic perturbations, and is subject to darkening due to neutron and gamma radiation.

A frequency-offset Michaelson optical interferometer configuration compensates the temperature and ambient acoustic-related phase variations down to the femtosecond level drifts over days, similar to one developed for the Atacama Large Millimeter Array radio telescope.[1]. As this system stabilizes the phase length of an optical fiber, the difference of the temperature dependence of the phase and group velocity of a long fiber so the group velocity variations may be determined from the phase velocity variation and corrected for.

\section{PHASE STABILIZER}

A frequency-offset Michaelson interferometer launches a $1530 \mathrm{~nm}$ optical signal from a CW laser with at least a $25 \mathrm{~km}$ coherence length along the fiber to be stabilized. In our case the fiber comprises $2 \mathrm{~km}$ of SMF- 28 single-mode fiber on spools located in a temperature-controlled room in series with another $2 \mathrm{~km}$ of SMF-28 fiber routed among

\footnotetext{
* This work is supported by the Director, Office of Science, High Energy Physics, U.S. Dept. of Energy under Contract no. DE-AC02$05 \mathrm{CH} 1121$
}

several buildings at LBNL, including under-road crossings and fiber patch panels.

At the far end, an acousto-optical modulator (AOM), driven by a $55 \mathrm{MHz}$ reference signal upshifts the optical frequency by $55 \mathrm{MHz}$, followed by a Faraday rotator mirror. The optical carrier returns through the AOM for another $55 \mathrm{MHz}$ upshift and back through the fiber to the sending point. The return signal is optically mixed with a sample of the original laser signal, and the resulting 110 $\mathrm{MHz}$ beat detected with a photodiode and phase compared to the $110 \mathrm{MHz}$ reference, which also, through a divider, provides the $55 \mathrm{MHz}$ to the AOM. The resulting error signal drives a phase modulator which consists of a piezo fiber stretcher in series with a motor-driven optical gap. Up to $600 \mathrm{psec}$ of time shift in the fiber can be accommodated, and the loop bandwidth, limited by both a $19 \mathrm{kHz}$ resonance in the piezo fiber stretcher and the system delay time of the $4 \mathrm{~km}$ fiber itself, is about $3 \mathrm{kHz}$.

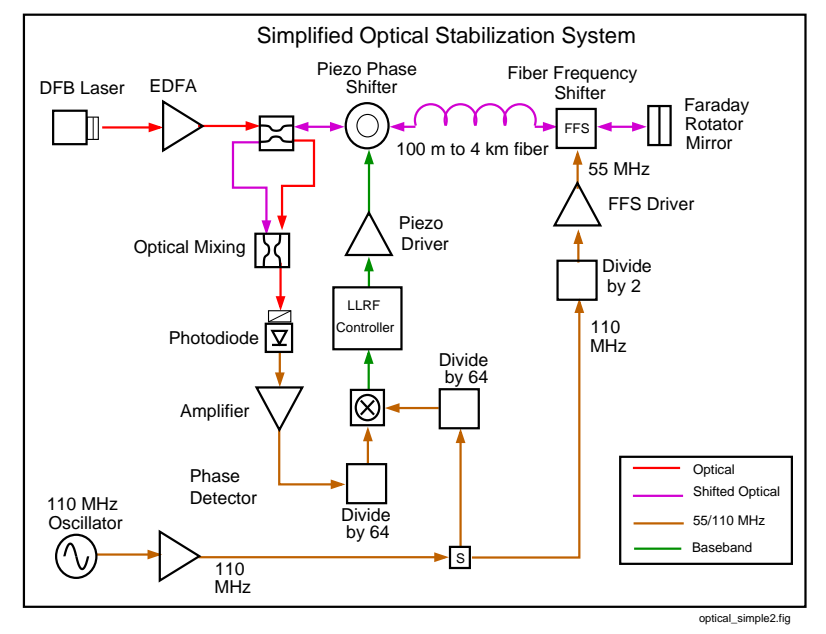

Figure 1: Simplified Optical Stabilization System

A fast perturbation of the phase, outside of the bandwidth of the control system, could result in the jumping of one or more optical fringes, as the error correction voltage is modulo $2 \pi$ of the optical phase. The range of the phase detector is extended a factor of 64 with the inclusion of by-64 frequency dividers in each input branch of the ring-diode phase detector. The output of each divider is a square wave, resulting in an output of the phase detector linearly proportional to the phase difference of the two inputs. The dividers increased the tolerance of the system to phase jumps by a factor of 64 and has resulted in no detectable fringe jumps.

The frequency offset technique rejects intermediate reflections along the fiber, as they would not have the 110 
$\mathrm{MHz}$ offset and would not produce a beat note. The Faraday mirror insures that the polarization between the return optical signal and the local laser sample remain fixed so a beat note may be reliably obtained. Figure 1 shows a simplified diagram of the phase stabilizer.

The frequency-offset approach offers a large leverage over stabilization in the RF domain. As the offset process is equivalent to a heterodyning process in which changes in optical phase translate to identical changes in the $110 \mathrm{MHz}$ beat signal. One degree of phase error in the RF domain translate to 1 degree of phase error at the $1530 \mathrm{~nm}$ laser frequency, or an equivalent time shift of 0.028 attoseconds. This six-order-of-magnitude leverage allows the use of offthe shelf RF components that do not need precise temperature regulation. Only the local arm of the fiber-based interferometer must be temperature controlled. These small components are in thermal isolation boxes kept to a temperature stability of better than $0.1 \mathrm{C}$.

\section{LASER FREQUENCY STABILIZATION}

The wavelength of the CW laser line provides the reference for the phase stabilization of the transmission fiber, as the interferometer maintains a constant number of fringes over the length of the fiber. The laser wavelength is locked to a molecular absorption line of acetylene $\left(\mathrm{C}_{2} \mathrm{H}_{2}\right)$ by a Pound-Drever-Hall (PDH) stabilizer. A sample of the laser output is frequency modulated by a Mach-Zehnder modulator at $500 \mathrm{MHz}$ and passed through a low-pressure (50 Torr) acetylene cell. The resulting amplitude modulation as the laser signal frequency interrogates the peak of the absorption band is coherently phase detected with the 500 $\mathrm{MHz}$ modulation signal and fed back to the frequencydetermining components of the laser itself. We estimate the laser wavelength is held to better than 1 part on $10^{9}$.

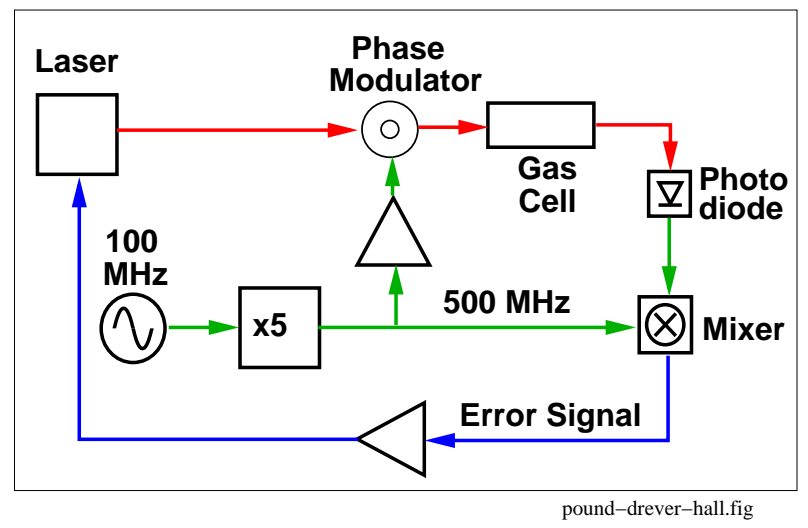

Figure 2: Pound-Drever-Hall Laser Stabilizer

Figure 2 shows a simplified diagram of the PDH laser wavelength stabilizer.

\section{FIBER PHASE STABILIZATION PERFORMANCE}

In use, a star configuration with a centrally-located clock a fan-out provides an optical carrier for each receiver located at the end of each individual fiber. The phase of the recovered optical carrier at the end of each fiber is maintained within the drift specifications of the fiber length stabilization loops.

To measure the common-mode optical phase stability, we set up two independently stabilized fibers using a common $1530 \mathrm{~nm} \mathrm{CW}$ laser. One fiber is a $3 \mathrm{~km}$ length of SPF28 , comprising $2 \mathrm{~km}$ of fiber laid between several buildings on the LBNL site in series with $1 \mathrm{~km}$ wound on a spool in a temperature-controlled laboratory environment.

3 km Fiber Length Correction vs. Time

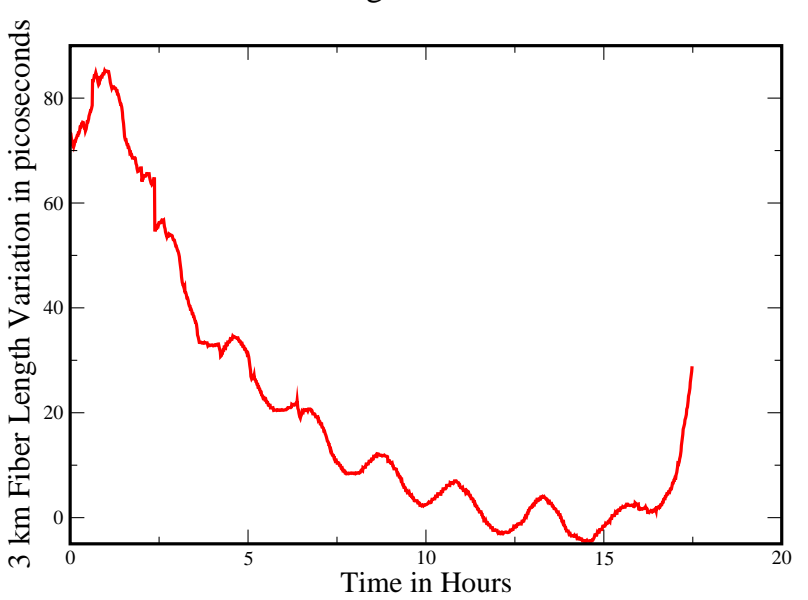

Figure 3: Phase Velocity Variation vs. External Temperature Variation

Differential Phase Difference of Two Fiber Channels

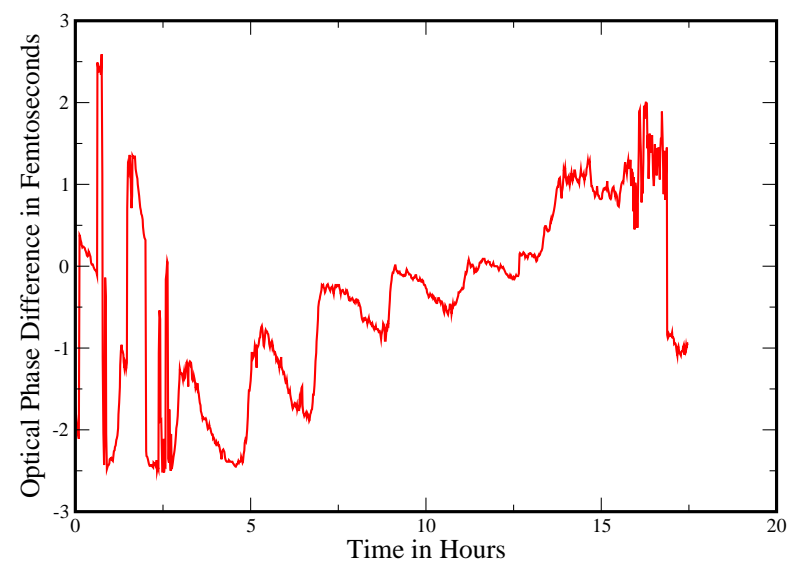

Figure 4: Phase Difference Between Two Stabilized Fibers

The other stabilized fiber loop is a short, 2 meter loop, maximizing the difference between the two independently stabilized fibers. (Stabilizing two fibers of different lengths reduces the possibility of common-mode coherence.) 
The optical phase difference between the two independently stabilized fibers is a measure of the operational stability and drift of the system. The AOMs in each loop are arranged to up-convert in one loop and down-convert by 55 $\mathrm{MHz}$ in the other, so an optical sample taken at the far end of each fiber will have a frequency difference of $110 \mathrm{MHz}$, which can be compared directly to the $110 \mathrm{MHz}$ reference oscillator.

Phase Delay vs Time

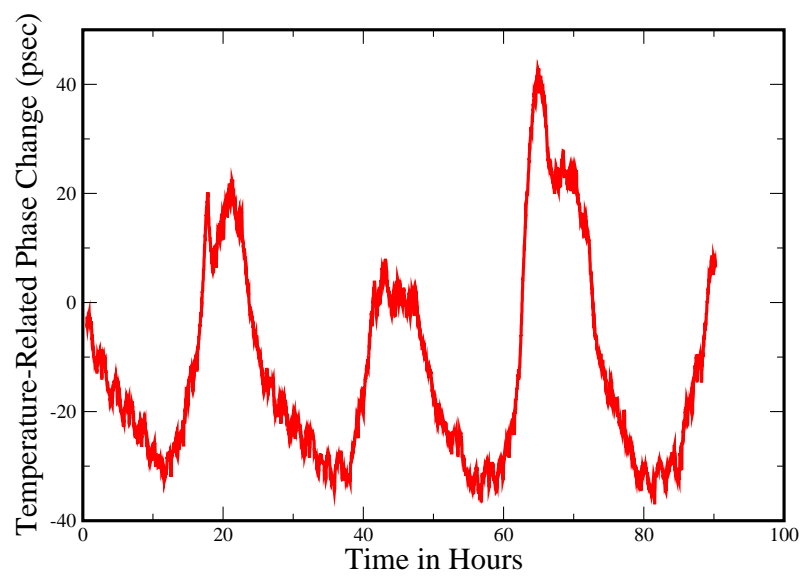

Figure 5: Temperature-related Phase Shift of $2 \mathrm{~km}$ Fiber

Predicted and Measured Group Delay Difference

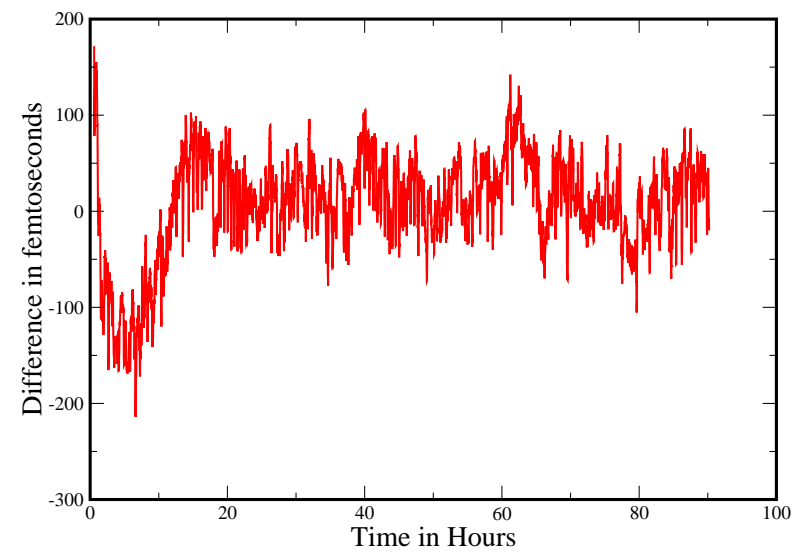

Figure 6: Difference (fsec) Between Predicted and Measured Group Delay

This technique constrains the RF phase variations of the stabilized fiber. When modulating RF signals on the fiber, the temperature variation of the group velocity must be stabilized.

Figure 3 shows the correction applied to the $3 \mathrm{~km}$ fiber loop over an 18 hour period, showing an 85 psec variation, mainly due to the diurnal outdoor temperature variation. Figure 4 shows the differential variation of optical phase (1 fringe of $1530 \mathrm{~nm}$ is equal to $5.1 \mathrm{fsec}$ ) between the long and the short stabilized fiber channels over the same time interval. The peak-to-peak variation is about 1 fringe (5.1 fsec). (The phase detector records modulo 2 pi, so a time variation of less than $-2.55 \mathrm{fsec}$ is wrapped around to +2.55 fsec, evident in the first two hours of data.)

The drift over the 18 hour interval is about $3.5 \mathrm{fsec}$, or $0.21 \mathrm{fsec} /$ hour. The hourly cyclic variations are due to the cycling of the laboratory room temperature of $1 \mathrm{C}$.

\section{GROUP VELOCITY CORRECTION}

To measure the ratio of the temperature dependence of the phase to group velocity, a $2.8 \mathrm{GHz} \mathrm{RF}$ signal is amplitude-modulated onto the output of the CW laser which is then passed through the system described above. A sample of the modulated optical carrier at the far end of $2 \mathrm{~km}$ of phase stabilized fiber is sampled and detected with a photodiode. The phase change of the transmitted $2.8 \mathrm{GHz} \mathrm{RF}$ is measured and correlated with the change of the phase velocity as the fiber undergoes the ambient temperature changes as in the dual-fiber experiment.

Figure 5 shows the variation of phase delay in $2 \mathrm{~km}$ of fiber subjected to diurnal temperature variation over 90 hours. Figure 6 shows the difference between measured and predicted group delay over the same time period. The predicted group velocity variation over and above the phase velocity change is the sum of $1.65 \%$ of the measured phase velocity change (Figure 5 data) and a constant and monotonic drift of -6.5 femtoseconds/hour. This drift is not yet understood and is being investigated.

\section{SYNCHRONIZING MODE-LOCKED LASERS}

Having a method for high-stability transmission of phase information enables synchronization of repetitively pulsed lasers. We are developing a scheme which synchronizes two modelocked lasers by comparing two comb lines of each[2]. This method does not require either of the modelocked lasers be CEO stabilized. Here, two CW lasers are locked to two $5 \mathrm{THz}$-separated comb lines of a reference modelocked laser. The two frequencies are transmitted and independently phase stabilized using acousto-optics driven by IQ RF modulators along a single fiber. When received, the two frequencies are interferometrically compared with two comb lines of the controlled laser. At each frequency the $\mathrm{CW}$ and comb phases are subtracted, and then subtracted again between the two frequencies, to yield an error signal that drives the controlled lasers repetition rate. Thus the envelopes of the two lasers are synchronized. Even though phase delay and not group delay is stabilized in this scheme, group delay is implicitly accounted for by the method of phase subtraction. The result is the same as if the controlled laser was synchronized to the $5 \mathrm{THz}$ beat note between the two optical frequencies.

\section{REFERENCES}

[1] A. Wootten, The Atacama Large Millimeter Array (ALMA), Proc. SPIE Vol. 4837, p. 110 (2003). 
[2] R. Wilcox, J. Staples, Systems Design Concepts for Optical Synchronization in Accelerators, Paper FROAC05, PAC 2007, Albuquerque 